\title{
Design of a hybrid silicon-plasmonic co- propagating coupler operating close to coherent perfect absorption
}

Cite as: J. Appl. Phys. 119, 163103 (2016); https://doi.org/10.1063/1.4947279

Submitted: 19 January 2016 . Accepted: 10 April 2016 . Published Online: 25 April 2016

Simone Zanotto (D), and Andrea Melloni

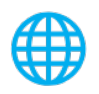

\section{ARTICLES YOU MAY BE INTERESTED IN}

Selective coherent perfect absorption in metamaterials

Applied Physics Letters 105, 201909 (2014); https://doi.org/10.1063/1.4902330

Coherent absorption of light by graphene and other optically conducting surfaces in realistic on-substrate configurations

APL Photonics 2, 016101 (2017); https://doi.org/10.1063/1.4967802

Coherent perfect absorption in an all-dielectric metasurface

Applied Physics Letters 108, 121901 (2016); https://doi.org/10.1063/1.4944635

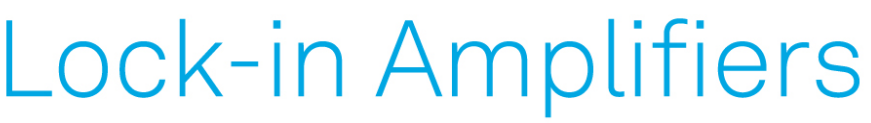

... and more, from DC to $600 \mathrm{MHz}$ Watch 


\title{
Design of a hybrid silicon-plasmonic co-propagating coupler operating close to coherent perfect absorption
}

\author{
Simone Zanotto ${ }^{\text {a) }}$ and Andrea Melloni \\ Dipartimento di Elettronica, Informazione e Bioingegneria, Politecnico di Milano, Piazza Leonardo da Vinci 32, \\ 20133 Milano, Italy
}

(Received 19 January 2016; accepted 10 April 2016; published online 25 April 2016)

\begin{abstract}
By hybrid integration of plasmonic and dielectric waveguide concepts, it is shown that nearly perfect coherent absorption can be achieved in a co-propagating coupler geometry. First, the operating principle of the proposed device is detailed in the context of a more general $2 \times 2$ lossy coupler formalism. Then, it is shown how to tune the device in a wide region of possible working points, its broadband operation, and the tolerance to fabrication uncertainties. Finally, a complete picture of the electromagnetic modes inside the hybrid structure is analyzed, shining light onto the potentials which the proposed device holds in view of classical and quantum signal processing, nonlinear optics, polarization control, and sensing. Published by AIP Publishing.

[http://dx.doi.org/10.1063/1.4947279]
\end{abstract}

\section{INTRODUCTION}

Losses occurring in optical systems are either strongly desired or carefully avoided, depending on the specific device under consideration. For instance, detectors and solar cells fall in the first category, whereas integrated optical elements such as waveguides and couplers usually belong to the second one. However, far from being an exhausted field of study, the analysis of absorption in linear optical systems recently attracted the community's attention, mainly in connection with the non-trivial phenomenon known as coherent perfect absorption (CPA). In essence, CPA consists of the complete absorption of two coherent beams by an otherwise partially absorbing sample or device. As long as monochromatic stationary waves are concerned, CPA has the remarkable property of being the time-reversal counterpart of a laser at threshold; ${ }^{1}$ even richer physical analogies with lasers beyond threshold occur when transient or chaotic optical fields are involved. ${ }^{2}$

While most of the studies about CPA focused upon structures working with free-space optical radiation, ${ }^{3-10}$ there are only few reports which treat the phenomenon within the integrated optics' framework, ${ }^{11,12}$ despite the integration of a CPA device on a scalable, on-chip platform could have a deep impact on different fields of optical science and technology. CPA is closely related to critical coupling, as already highlighted in Ref. 1, and critical coupling is a well established concept in integrated optics. ${ }^{13,14}$ However, CPA-and its precursor, coherent absorption (CA) —offers a further functionality, that is, the full optical control of the absorption. This feature can be exploited both for coherent processing of classical signals and for quantum optical computing purposes. ${ }^{15,16}$ On one hand, the CPAbased detection schemes for phase-keyed signals have been devised, ${ }^{17}$ while on the other many opportunities related to the statistics of individual photons are still to be explored.

\footnotetext{
${ }^{a}$ Present address: Istituto Nazionale di Ottica-CNR, and LENS, Via Nello Carrara 1, 50019 Sesto Fiorentino (FI), Italy.
}

Possible experiments generalizing those with cascades of non-lossy beam splitters ${ }^{18,19}$ could be envisaged, exploiting the apparent two-photon absorption in a linear medium which is enabled by CPA. ${ }^{20}$

Here, we propose, design, and analyze a co-propagating device operating close to CPA based on the hybrid integration of a plasmonic element into a $2 \times 2$ coupler implemented on the silicon-on-insulator platform. The co-propagating geometry is complementary to the counter-propagating one reported in Ref. 12, helping the optical designer to develop more complex projects. Our device expands the current library of hybrid devi$\mathrm{ces}^{21}{ }^{21}$ sharing with them the potentials for biosensing, nonlinear optics, ${ }^{22-25}$ and even for detection, if the hot carriers generated in the metal are collected through Schottky barriers. ${ }^{26-28}$

\section{DEVICE CONCEPT AND THEORETICAL FRAMEWORK}

The concept of a generic co-propagating CPA coupler is schematized in Figs. 1(a) and 1(b). Considering structures with a symmetry axis, two distinct possibilities exist. Either the system fully absorbs the symmetric input state or it absorbs the antisymmetric one. This is a consequence of the CPA condition, here written $\operatorname{det} T=0, T$ being the $2 \times 2$ coupler transfer matrix. In other words, one of the $T$-matrix eigenvalue is zero, and its eigenstate can be either the symmetric or the antisymmetric one. In addition, a common requirement (referred to as coherent perfect transparency, CPT) is that the second eigenvalue has unity modulus. This means that the input state orthogonal to the absorbed one is perfectly transmitted; this situation is also depicted in Figure 1. Notice that at this stage, the discussion is about the states of the coupler in its whole, not of the coupling region, and that there are, in principle, many routes to realize a CPA co-propagating coupler. Nonetheless, a closer analysis which will be discussed in detail in the following reveals that a proper CPA is out of reach of a co-propagating coupler, at least when it is treated under the coupled-mode approximation. Still, a device 


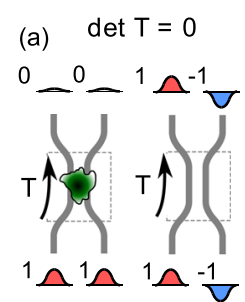

(b)
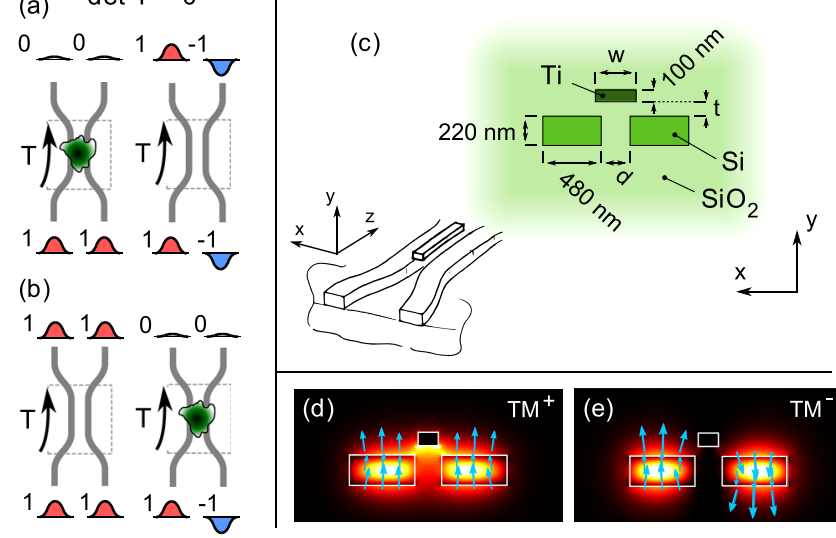

FIG. 1. Panels (a) and (b): schematical operation of an axisymmetric copropagating CPA coupler. With the symmetry constraint, CPA can either occur for in-phase or out-of-phase inputs. Panel (c): a quasi-CPA coupler implemented through hybrid integration of a metal component with silicon photonic coupled waveguides. Its operating principle is the difference between the field distribution among even and odd TM supermodes [panels (d) and (e)]. The large extinction of the $\mathrm{TM}^{+}$supermode leads to a situation such that of panel (a).

operating arbitrarily close to CPA can be simply synthesized by engineering the absorption of the symmetric and antisymmetric supermodes of the coupling region, by properly modifying an ordinary directional coupler. A geometry which implements this idea is depicted in Fig. 1(c). Two standard $220 \times 480 \mathrm{~nm}$ silicon waveguides are buried into a $\mathrm{SiO}_{2}$ cladding, and a titanium stripe is placed on the axis symmetry of the coupler, vertically spaced by $t$ from the waveguide's top plane. Titanium is chosen as a high-loss metal compatible with silicon photonics processing. The waveguides are separated by $d$, and the metal stripe width is $w$. A thorough analysis of the effect of the parameters will be detailed later.

Figs. 1(d) and 1(e) depict the computed symmetric and antisymmetric (quasi-) transverse-magnetic supermodes of the coupling region, labeled, respectively, $\mathrm{TM}^{+}$and $\mathrm{TM}^{-}$. Ordinary mode analysis performed with a commercial finiteelement mode solver (COMSOL) has been employed. As it can be directly observed by the color map, which represents the $z$-component of the Poynting vector, the $\mathrm{TM}^{+}$mode shows some energy flux in close vicinity of the metal stripe. This eventually ends up in a large extinction coefficient for that mode. Instead, in the $\mathrm{TM}^{-}$supermode, which must fulfil field antisymmetry with respect to the vertical symmetry axis, there is no electromagnetic energy guided in close vicinity of the metal stripe, and hence, a lower absorption is expected. The complex propagation constants of the coupler supermodes $\beta^{ \pm}$are the key quantities entering the lossy coupler $T$-matrix and the CPA/CPT conditions. The $\beta^{ \pm}$are defined in terms of the complex effective refractive indices of the coupler supermodes $n_{\text {eff }}^{ \pm}$as $\beta^{ \pm}=2 \pi\left(\operatorname{Re} n_{\text {eff }}^{ \pm}+i \operatorname{Im} n_{\text {eff }}^{ \pm}\right) / \lambda_{0}, \lambda_{0}$ being the vacuum wavelength. Neglecting any spurious effect (i.e., reflections, radiation, and imperfect modal overlap) at the transition regions, the usual coupled-mode analysis leads to

$$
T=\left(\begin{array}{cc}
t_{11} & t_{21} \\
t_{12} & t_{22}
\end{array}\right) ; \quad \begin{aligned}
& t_{11}=t_{22}=\left(e^{i \beta^{+} L}+e^{i \beta^{-} L}\right) / 2 \\
& t_{12}=t_{21}=\left(e^{i \beta^{+} L}-e^{i \beta^{-} L}\right) / 2
\end{aligned},
$$

where $L$ is the length of the coupling region. Simple algebra leads to $\operatorname{det} T=e^{i \beta^{+} L} e^{i \beta^{-} L}$. From this expression, it turns out that a proper coherent perfect absorption condition cannot be met. However, if either $\operatorname{Im} \beta^{+} L$ or $\operatorname{Im} \beta^{-} L$ is large, the system is close to CPA. Better yet, if $\operatorname{Im} \beta^{+} \gg \operatorname{Im} \beta^{-}$(or the reverse), the system is both close to CPA and to CPT. Indeed, up to a global phase, under this condition, the transmission matrix approaches the limit

$$
T \rightarrow\left(\begin{array}{cc}
1 / 2 & \pm 1 / 2 \\
\pm 1 / 2 & 1 / 2
\end{array}\right)
$$

where the sign is determined by the relative magnitude of $\operatorname{Im} \beta^{ \pm}$. Referring to the specific device geometry analyzed in the present work, $\operatorname{Im} \beta^{+} \gg \operatorname{Im} \beta^{-}$, and the minus sign applies. Notice that, when a single arm of the coupler is excited, the output signals are either in phase or out of phase by $\pi$, as opposed to ordinary lossless directional couplers where a $\pi / 4$ dephasing is observed.

To quantify how an engineered lossy coupler is close to CPA and CPT, it is useful to rely on two quantities, extinction ratio (ER) and insertion loss (IL). In this framework, they are defined as $\mathrm{IL}=-10 \log _{10}\left(I_{\text {out;max }} / I_{\text {in }}\right)$ and $\mathrm{ER}=-10 \log _{10}$ $\left(I_{\text {out;min }} / I_{\text {out;max }}\right)$, where $I_{\text {out;min,max }}$ are the total minimum and maximum intensities available at the output arms of the coupler. Minimum and maximum can be reached by sweeping the relative phase of the inputs; notice that in the $2 \times 2$ axisymmetric configuration, these extrema are reached when signals of equal intensity $I_{\text {in }} / 2$ are employed as inputs (balanced inputs; the behaviour of CA devices without axial symmetry are discussed in Ref. 29).

Insertion loss and extinction ratio can be interpreted both from an internal and an external point of view. In the first case, the focus is on the energy available inside the coupler, which can be harnessed, for instance, for heating, for detection, or for mechanical motion. ${ }^{30}$ In the second case, the main point is about the energy which is delivered at the output ports, which can be subsequently detected or rerouted into a forthcoming circuit. In both cases, IL should be as small as possible, while ER should be maximized. Clearly, IL and ER are connected to the T-matrix elements: when the generic form for the T-matrix is considered, one gets $I_{\text {out;max }}=2\left|t_{11} \pm t_{12}\right|^{2}$, where the sign is determined by whether the coupler has a symmetric or antisymmetric CA. ${ }^{31}$ When instead the co-propagating CA coupler based on differential losses between supermodes is analyzed, in the case $\operatorname{Im} \beta^{+}>\operatorname{Im} \beta^{-}$, the expressions $\mathrm{IL}=20 \log _{10} \exp \left(\operatorname{Im}\left(\beta^{-}\right) L\right)$ and $\mathrm{ER}=20 \log _{10} \exp \left(\operatorname{Im}\left(\beta^{+}-\beta^{-}\right) L\right)$ are obtained. The linear dependence of IL and ER upon the coupler length $L$ pushed us to redefine those quantities according to $\mathrm{IL} \rightarrow \mathrm{IL} / L$, $\mathrm{ER} \rightarrow \mathrm{ER} / L$; in the following, IL and ER will refer to the latter definition and will be expressed in $\mathrm{dB} / \mu \mathrm{m}$. It should be noticed that the ratio IL/ER is unaffected by this substitution. The forthcoming analysis will also focus on this ratio, since it is a quantity which only depends on the waveguide design and not on the choice of a specific coupler length.

\section{PARAMETRIC ANALYSIS}

As the geometrical parameters and the operating wavelength are varied, the device of Fig. 1(c) shows different values 
of IL and ER. For instance, if $t=70 \mathrm{~nm}, w=150 \mathrm{~nm}$, $d=200 \mathrm{~nm}$, and $\lambda=1.55 \mu \mathrm{m}$, one obtains $\mathrm{IL}=0.11 \mathrm{~dB} / \mu \mathrm{m}$ and $\mathrm{ER}=1.23 \mathrm{~dB} / \mu \mathrm{m}$. Hence, devices with lengths of the order of tens of microns can implement a satisfactory extinction factor.

When the parameters $w, t$, and $d$ are varied, the results reported in Fig. 2 are obtained: in the left column, $t$ and $w$ are varied while $d=200 \mathrm{~nm}$ is kept fixed; in the right column, instead, $d$ and $w$ are swept while $t=70 \mathrm{~nm}$ is kept constant. The operating wavelength is fixed at $1.55 \mu \mathrm{m}$. In each column, the dashed lines highlight the value of the parameter which is kept constant in the other column; the black dots, instead, represents the parameters which will be employed in Fig. 3. The rows of Fig. 2 group insertion loss, extinction ratio, and the "figure of merit" ER/IL. Analyzing Fig. 2, three main features stand out. First, insertion loss is strongly affected by $w$ and weakly by $t$ and $d$. Values as small as $0.02 \mathrm{~dB} / \mu \mathrm{m}$ can be obtained for $w \simeq 100 \mathrm{~nm}, d \simeq 400 \mathrm{~nm}$. Second, the effect on the extinction ratio is mostly ascribed to the waveguide spacing $d$, which, when brought down to $d \simeq 100 \mathrm{~nm}$, leads to ER's almost as large as $3 \mathrm{~dB} / \mu \mathrm{m}$. Third, the combined effect of the above leads to an overall insensitivity of the ratio ER/IL upon variations of both $t$ and $d$, whereas it is largely affected by $w$. These features can be exploited to fit the CA coupler to the desired working point, as well as to cope with the tolerance issues inherent in the fabrication processes.

It will now be shown that the device is also robust with respect to wavelength and size perturbations. The data reported in Figs. 3(a) and 3(b) are obtained for fixed values of the physical dimensions (reported in the schematic), while
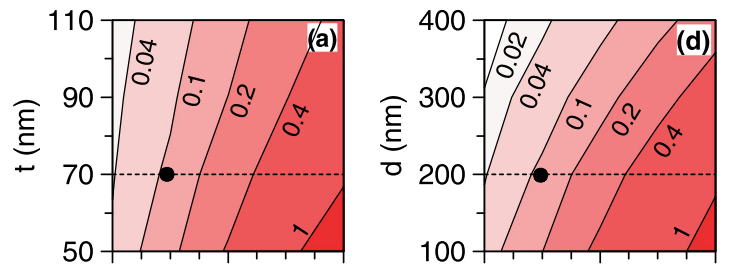

IL $(\mathrm{dB} / \mu \mathrm{m})$
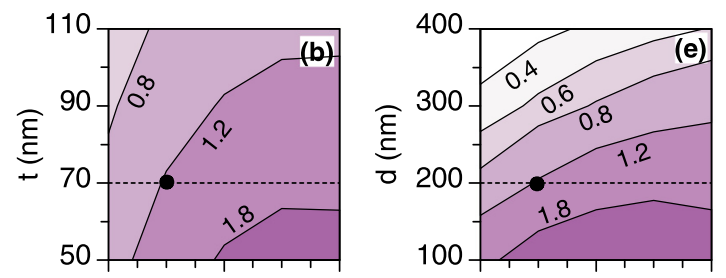

$(\mathrm{dB} / \mu \mathrm{m})$
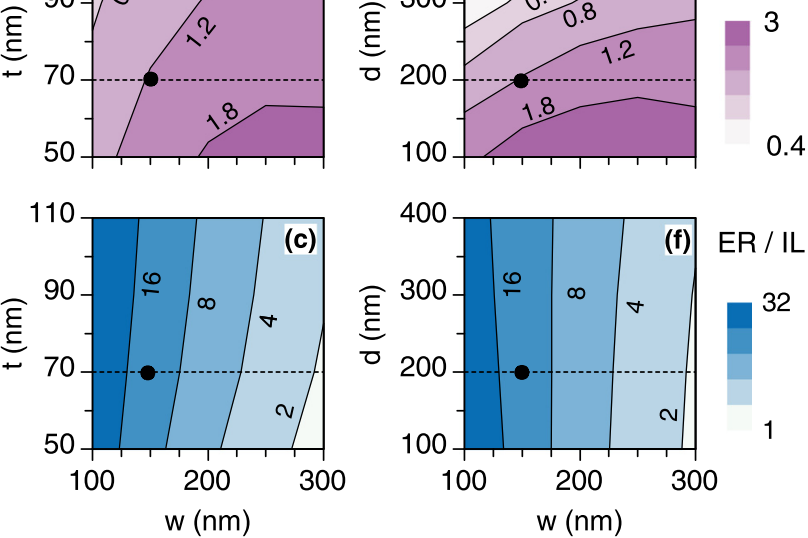

FIG. 2. Dependence of the coherent absorption coupler key parameters insertion loss and extinction ratio (IL and ER) with respect to the geometrical parameters $t, w$, and $d$. In the left panels, $d=200 \mathrm{~nm}$, while in the right ones, $t=70 \mathrm{~nm}$. IL and ER can be tailored by properly tuning $t, w$, and $d$.
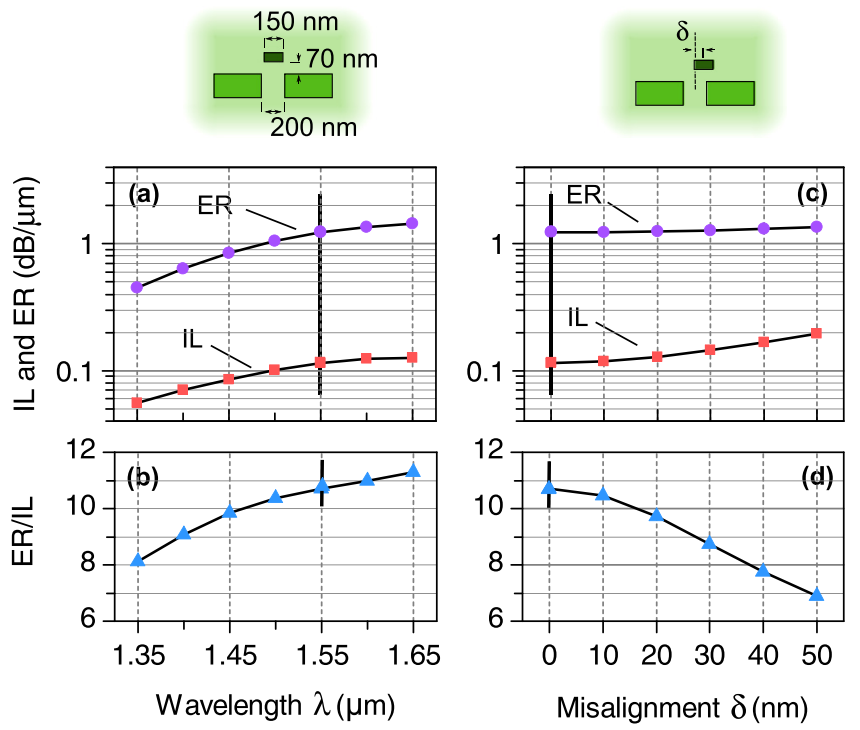

FIG. 3. Panels (a) and (b): insertion loss (IL) and extinction ratio (ER) of a hybrid coupler, as a function of the wavelength, for the geometrical parameters reported in the schematics. Large ER and small IL means that the device is close to CPA and CPT (see text). IL and ER are not strongly affected by the wavelength. Even smaller is the effect on the ratio ER/IL. Similar observations follow from the analysis of the tolerance with respect to the metal stripe misalignment, panels (c) and (d).

the wavelength is swept within a wide band relevant for data- and telecommunication purposes. The insertion loss increases with the wavelength, from about $0.06 \mathrm{~dB} / \mu \mathrm{m}$ at $1350 \mathrm{~nm}$ to $0.13 \mathrm{~dB} / \mu \mathrm{m}$ at $1650 \mathrm{~nm}$. Meanwhile, the extinction ratio increases from $0.45 \mathrm{~dB} / \mu \mathrm{m}$ at $1350 \mathrm{~nm}$ to $1.4 \mathrm{~dB} / \mu \mathrm{m}$ at $1650 \mathrm{~nm}$ : in the whole band under analysis, ER is almost ten times larger (in dB scale) with respect to IL (see Fig. 3(a)).

When the misalignment $\delta$ of the metal stripe is instead considered as the tolerance parameter, the data in Figs. 3(c) and 3(d) are obtained. Here, the wavelength is fixed at $1550 \mathrm{~nm}$, and the component sizes are the same as above. As it can be expected, the performance metric ER/IL decreases when $\delta$ increases; however, even for the quite large value $\delta=50 \mathrm{~nm}, \mathrm{ER} / \mathrm{IL}$ is as large as 7 units. The main reason is the increase in IL, while ER is essentially unaffected by the misalignment. While the tolerance of the CA coupler with respect to the wavelength could have been expected, the device being not based on a resonance mechanism, the robustness with respect to the misalignment is more surprising. Indeed, symmetry is the operating principle of the device, and its breaking could have been suspected of a significant degradation of the performance.

\section{FULL MODAL ANALYSIS}

This section is devoted to a full modal analysis of the hybrid coupler, which gives further insights into the operating potentials of the proposed structure, possibly in view of applications not strictly related to the coherent perfect absorption mechanism. Fig. 4 represents a modal map of the hybrid coupler: the real part of the supermodes' effective refractive index is plotted on the $x$-axis, while the imaginary part is plotted on the $y$-axis. The parameters $w=150 \mathrm{~nm}, t=70 \mathrm{~nm}$, and $d=200 \mathrm{~nm}$ have been chosen, while the wavelength is 


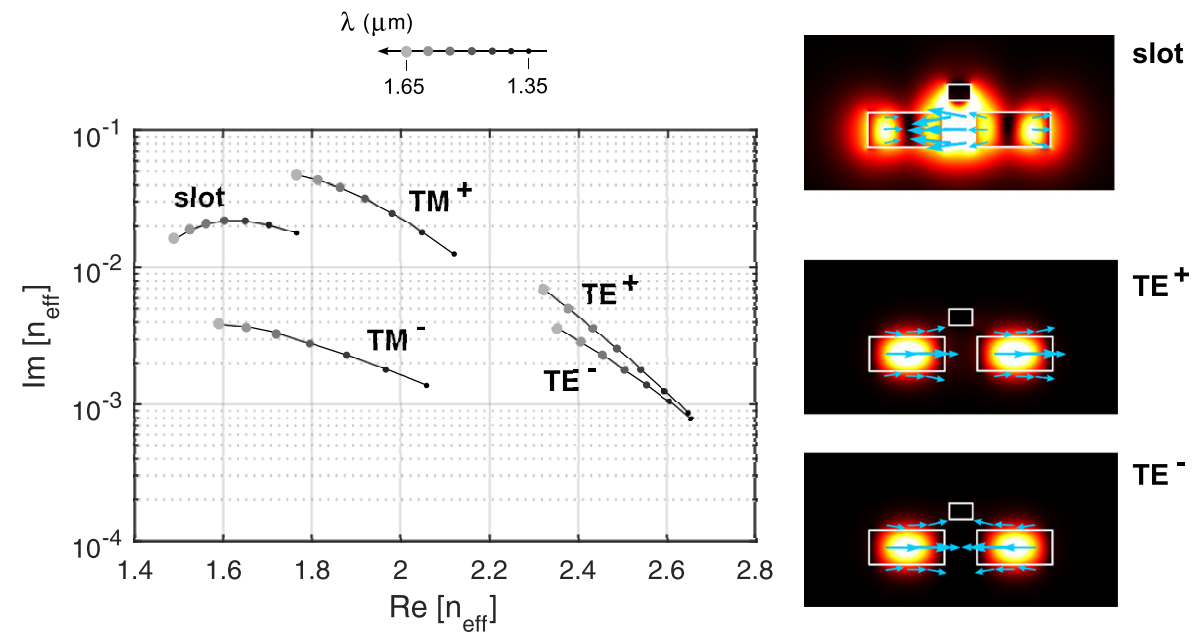

FIG. 4. Modal map in the complex effective refractive index plane for the hybrid silicon-plasmonic coupler. The TE and TM modes appear as pairs of supermodes with opposite symmetries, while a slot-like mode (hybridized with a plasmonic component) exists as a singlet. All the modes exist in the $(1.35,1.65) \mu \mathrm{m}$ wavelength range, without mixing or crossing effects. The color maps represent the out-of-plane component of the Poynting vector, while the blue arrows represent the modal transverse electric field. The strong difference between $\operatorname{Im} n_{\text {eff }}$ for the TM modes is at the hearth of the CPA operation.

swept from 1.35 to $1.65 \mu \mathrm{m}$ (i.e., the same as in Figs. 3(a) and 3(b)). The modes are grouped according to their symmetry and polarization: the field profiles of $\mathrm{TM}^{ \pm}$modes are already represented in Figs. 1(d) and 1(e), while those of the other modes are reported here to the right of the graph. This representation immediately reveals several features of the studied structure. First, within the considered wavelength range, the complex effective index of each mode evolves continuously, indicating that no mode crossing and mixing occur. Second, the $\mathrm{TM}^{ \pm}$ modes exhibit a large splitting between $\operatorname{Im} n_{\text {eff }}$. This is a graphical way of understanding the co-propagating CA effect, since, as it was highlighted above, a condition which implies quasi$\mathrm{CPA}$ is that $\operatorname{Im} \beta^{+} \gg \operatorname{Im} \beta^{-}$. Notice that, instead, the $\mathrm{TE}^{ \pm}$ modes have a small splitting between $\operatorname{Im} n_{\text {eff }}$ : in essence, the hybrid coupler acts as a polarization-sensitive CA device. Third, in addition to the TE and TM modes, the device exhibits a peculiar dielectric slot-surface plasmon hybrid mode, which exists in a single symmetry configuration and which cannot be thereby exploited in view of CA. However, thanks to the strong field confinement in the cladding region and in the vicinity of the metal stripe, this mode could be harnessed for switching purposes in nonlinear materials, opening up the perspective for further functionalities.

\section{CONCLUSIONS}

In conclusion, the design of an integrated optical component operating close to coherent perfect absorption and transparency (CPA and CPT) is presented. The layout exploits hybridization of dielectric and plasmonic modes in a co-propagating coupler realized on the silicon photonic platform. By harnessing the tight field confinement together with the metal losses, a large extinction ratio and small insertion losses can be devised. A numeric analysis revealed that different working point can be properly set by tuning certain structure parameters, and that the structure is tolerant to fabrication uncertainties. The co-propagating coherent absorption device, whose general behaviour in terms of $2 \times 2$ transfer matrices is also discussed, can be included in complex networks to elaborate classical and optical signals, offering new functionalities such as signal demodulation and apparent single-photon nonlinearities in a single, smallfootprint building block.

${ }^{1}$ Y. D. Chong, L. Ge, H. Cao, and A. D. Stone, Phys. Rev. Lett. 105, 053901 (2010).

${ }^{2}$ S. Longhi and G. Della Valle, Phys. Rev. A 85, 053838 (2012).

${ }^{3}$ W. Wan, Y. Chong, L. Ge, H. Noh, A. D. Stone, and H. Cao, Science 331, 889 (2011).

${ }^{4}$ H. Noh, Y. Chong, A. D. Stone, and H. Cao, Phys. Rev. Lett. 108, 186805 (2012).

${ }^{5}$ S. Feng and K. Halterman, Phys. Rev. B 86, 165103 (2012).

${ }^{6}$ M. Pu, Q. Feng, M. Wang, C. Hu, C. Huang, X. Ma, Z. Zhao, C. Wang, and X. Luo, Opt. Express 20, 2246 (2012).

${ }^{7}$ J. W. Yoon, G. M. Koh, S. H. Song, and R. Magnusson, Phys. Rev. Lett. 109, 257402 (2012).

${ }^{8}$ J. Zhang, C. Guo, K. Liu, Z. Zhu, W. Ye, X. Yuan, and S. Qin, Opt. Express 22, 12524 (2014).

${ }^{9}$ S. M. Rao, J. J. F. Heitz, T. Roger, N. Westerberg, and D. Faccio, Opt. Lett. 39, 5345 (2014).

${ }^{10}$ T. Roger, S. Vezzoli, E. Bolduc, J. Valente, J. J. Heitz, J. Jeffers, C. Soci, J. Leach, C. Couteau, N. I. Zheludev et al., Nat. Commun. 6, 7031 (2015).

${ }^{11}$ R. R. Grote, J. B. Driscoll, and R. M. Osgood, Opt. Lett. 38, 3001 (2013).

${ }^{12}$ R. Bruck and O. L. Muskens, Opt. Express 21, 27652 (2013).

${ }^{13}$ M. Cai, O. Painter, and K. J. Vahala, Phys. Rev. Lett. 85, 74 (2000).

${ }^{14}$ A. Yariv, IEEE Photonics Technol. Lett. 14, 483 (2002).

${ }^{15}$ N. P. De Leon, M. D. Lukin, and H. Park, IEEE J. Sel. Top. Quantum Electron. 18, 1781 (2012).

${ }^{16}$ R. W. Heeres, L. P. Kouwenhoven, and V. Zwiller, Nat. Nanotechnol. 8, 719 (2013); e-print arXiv:1309.6942.

${ }^{17}$ R. R. Grote, J. M. Rothenberg, J. B. Driscoll, and R. M. Osgood, in CLEO: Science and Innovations (Optical Society of America, 2015), $\mathrm{p}$. STh4F-1.

${ }^{18}$ M. Tillmann, B. Dakić, R. Heilmann, S. Nolte, A. Szameit, and P. Walther, Nat. Photonics 7, 540 (2013).

${ }^{19}$ N. Spagnolo, C. Vitelli, M. Bentivegna, D. J. Brod, A. Crespi, F. Flamini, S. Giacomini, G. Milani, R. Ramponi, P. Mataloni et al., Nat. Photonics 8, 615 (2014).

${ }^{20}$ S. M. Barnett, J. Jeffers, A. Gatti, and R. Loudon, Phys. Rev. A 57, 2134 (1998).

${ }^{21}$ M. Z. Alam, J. S. Aitchison, and M. Mojahedi, Laser Photonics Rev. 8, 394 (2014).

${ }^{22}$ M. W. Klein, C. Enkrich, M. Wegener, and S. Linden, Science 313, 502 (2006).

${ }^{23}$ W. Cai, A. P. Vasudev, and M. L. Brongersma, Science 333, 1720 (2011). 
${ }^{24}$ S. Sederberg and A. Y. Elezzabi, Phys. Rev. Lett. 114, 227401 (2015).

${ }^{25}$ N. Segal, S. Keren-Zur, N. Hendler, and T. Ellenbogen, Nat. Photonics 9 , 180 (2015).

${ }^{26}$ M. W. Knight, H. Sobhani, P. Nordlander, and N. J. Halas, Science 332, 702 (2011).

${ }^{27}$ M. L. Brongersma, N. J. Halas, and P. Nordlander, Nat. Nanotechnol. 10, 25 (2015).
${ }^{28}$ F. P. García de Arquer, A. Mihi, and G. Konstantatos, ACS Photonics 2, 950 (2015).

${ }^{29}$ L. Baldacci, S. Zanotto, G. Biasiol, L. Sorba, and A. Tredicucci, Opt. Express 23, 9202 (2015).

${ }^{30}$ M. A. Taylor, M. Waleed, A. B. Stilgoe, H. Rubinsztein-Dunlop, and W. P. Bowen, Nat. Photonics 9, 669 (2015).

${ }^{31}$ Note that symmetry and reciprocity imply $t_{11}=t_{22}$ and $t_{12}=t_{21}$. 